\title{
Editorial
}

\section{Cardiovascular risks and outcomes: ethnic variations in hypertensive patients}

Epidemiological studies of international variations in disease incidence have helped to generate and test hypotheses about the relative importance of genetic, environmental, social, and cultural factors in causing cardiovascular diseases. ${ }^{1}$ Studies of racial and ethnic variation within countries have also contributed in advancing such understanding. Indian, Bangladeshi, and Pakistani people born on the Indian subcontinent (henceforth called South Asian) but living in England and Wales have a 40-50\% higher mortality from coronary heart disease than the population average. ${ }^{2}{ }^{3}$ The prevailing hypothesis explaining this excess is the insulin resistance hypothesis. Coronary heart disease, paradoxically, is comparatively low among Afro-Caribbeans, ${ }^{4}$ who share with South Asians a high prevalence of insulin resistance.

Another paradox concerns hypertension, one of the major coronary risk factors, as ethnic variations in blood pressure do not parallel variations in coronary heart disease. Prevalence of hypertension among the AfroCaribbean and African populations is high, but their risk of death from coronary heart disease is low. ${ }^{4}$ There is conflicting evidence over whether hypertension is more common in South Asian groups. One study found diastolic blood pressure among South Asian (predominantly Punjabi) men in Glasgow was higher than in the general population, but this was not so for South Asian women, or for systolic blood pressure in South Asian men. ${ }^{6}$ South Asian groups in Newcastle, particularly Bangladeshis, were found to have lower blood pressure than Europeans. ${ }^{7}$ Paradoxically Bangladeshis have the highest mortality from coronary heart disease. ${ }^{3}$ These and many other paradoxes require cohort studies in order to be resolved; therefore the recent article by Khattar and colleagues is of special interest. $^{8}$

\section{Morbidity and mortality in a cohort of hypertensive patients}

Khattar and colleagues compared the mortality and morbidity among white $(n=528)$, South Asian $(n=106)$, and Afro-Caribbean $(n=54)$ patients investigated for essential hypertension between 1979 and 1993 in one hospital in England. ${ }^{8}$ Those with raised blood pressure underwent 24 hour ambulatory, intra-arterial blood pressure monitoring. Between 1994 and 1996, the investigators followed up patients, reassessed cardiovascular risk factors, and collected information on health outcomes. For patients who had died death certificates and medical records were examined. Outcome events were classified as noncardiovascular death, coronary events, cerebrovascular events, and peripheral vascular death. Coronary events included non-fatal myocardial infarction, coronary artery bypass graft surgery, and coronary angioplasty.

South Asians had higher all cause event rates than AfroCaribbeans and white patients, mainly from higher rates of coronary events. Age, sex, race, diabetes, previous history of cardiovascular disease, and 24 hour ambulatory systolic blood pressure (but not clinic blood pressure) were, in a multiple logistic regression model, independent predictors of a first event.
This is one of the few prospective studies of cardiovascular disease in UK ethnic groups. It has limitations including the use of the concept of race, inattention to population heterogeneity, small number of subjects (106 South Asians and 54 Afro-Caribbeans), age and sex differences between the populations, and incomplete (58\%) follow up. For patients with multiple events the events were added up, providing a crude outcome measure, but necessary because of the small numbers.

\section{Race, ethnicity, and population heterogeneity}

In studies of ethnic groups the term "race" is often used, as it is by Khattar and colleagues. This raises questions about the concepts guiding the study's classification of populations. Race as a biological concept is of limited use in epidemiology, for it is based primarily on physical features, which bear little relation to susceptibility to most diseases or to explaining genetic variations between populations. ${ }^{9}$ The concept of ethnicity is based upon several cultural and other features providing group identity. Khattar's paper calls South Asian, white and Afro-Caribbean groups races, when these are labels for broad ethnic groups. Differences between South Asian ethnic groups are important in the context of cardiovascular diseases, for the risk factor profiles differ dramatically, ${ }^{7}$ and disease outcomes differ too. $^{310}$

In Khattar's study, 73 out of 106 "South Asian" subjects are Gujaratis, who have a culture distinct from other South Asian groups-for example, language, food habits, and for many a history of living in East Africa. This study, predominantly of Gujaratis, is not necessarily generaliseable to other South Asian groups. This might explain why, unlike other studies, ${ }^{267}$ Khattar reported lower body mass index in South Asians than in whites and Afro-Caribbeans. Heterogeneity of study populations may be a general explanation for inconsistent results from studies of "South Asians" in Britain.

\section{Why coronary heart disease is more common in South Asians}

The study by Khattar potentially contributes to three broad explanations for the high rate of coronary heart disease in South Asian groups. First, they may have a higher prevalence of risk factors for coronary heart disease than populations of European origin. They have lower high density lipoprotein cholesterol, more diabetes, a greater lack of aerobic exercise, and greater central distribution of body fat. ${ }^{2}$ For low density lipoprotein (LDL) cholesterol, high blood pressure, and obesity, the picture is equivocal with differences between studies, communities, and sexes. Indian men, and all groups of South Asian women, have a comparatively low prevalence of smoking. Smoking prevalence in Pakistani men is similar to the whole population, and in Bangladeshi men it is much higher. South Asian groups have a higher prevalence of factors potentially leading to stress, particularly economic deprivation, social upheaval following migration, and racial discrimination. ${ }^{6}{ }^{1011}$ Khattar and colleagues do not report a sufficiently wide range of risk factors to examine this 
explanation critically, though in their population blood pressure and total cholesterol were marginally lower in South Asians, while diabetes was higher, and smoking about the same.

The second explanation is that the South Asian groups are more susceptible to coronary heart disease risk factors for environmental or genetic reasons. Khattar's study contributes to this theory, in that after adjustment for a range of risk factors, the rate of coronary heart disease among South Asians was higher compared to the white population. A potential non-genetic mechanism enhancing susceptibility in South Asians is a mismatch between early and later life metabolism. ${ }^{12}$ Dramatic changes in risk factors, including cholesterol, occur upon immigration ${ }^{13}$ and might present risks beyond that predicted from a single measure.

The third explanation is that South Asians have fewer competing causes of death at the age when coronary heart disease occurs. The fact that their cancer rates are comparatively low, ${ }^{4}$ and overall standardised mortality ratios lie around 100, provides some evidence for this as yet unexplored explanation. Khattar's study offers no support for this explanation.

\section{Conclusions}

High coronary heart disease rates in South Asians are still a mystery, needing more epidemiological research, especially population based cohort studies. Khattar's cohort study confirms that coronary heart disease risk is comparatively high among a predominantly Gujarati South Asian population with hypertension and points to enhanced susceptibility as an explanation.

Khattar and colleagues recommend intensive risk modification strategies. While the causal basis of ethnic variations in cardiovascular disease remains unclear, prevention and control strategies must be based on estab- lished risk factors, accounting for cultural and socioeconomic differences. Smoking, lack of exercise, hypertension, obesity, high fat diets, raised LDL cholesterol concentrations, and diabetes all contribute to coronary heart disease in ethnic minority groups and need to be addressed urgently. Ethnic minority communities must be well informed, and become involved in prevention and control campaigns.

RAJ BHOPAL SUSHMITA SENGUPTA-WIEBE

Public Health Sciences,

University of Edinburgh Medical School,

Teviot Place, Edinburgh EH8 9AG, UK

1 Tunstall-Pedoe H, Kuulasmaa K, Mahonen M, et al. Contribution of trends in survival in coronary event rates to changes in coronary heart disease mortality: 10-year results from 37 WHO MONICA project populations. Lancet 1999;353:1547-56.

2 McKeigue PM, Shah B, Marmot MG. Relation of central obesity and insulin resistance with high diabetes prevalence and cardio-vascular risk in South Asians. Lancet 1991;337:382-6.

3 Balarajan R. Ethnicity and variations in mortality from coronary heart disease. Health Trends 1996;28:45-51.

4 Wild S, McKeigue P. Cross sectional analysis of mortality by country of birth in England and Wales, 1970-92. BMF 1997;314:705-10.

5 Cappuccio FP. Ethnicity and cardiovascular risk: variations in people of African ancestry and South Asian origin. $\mathcal{F}$ Human Hypertens 1997;11:5716.

6 Williams R, Bhopal R, Hunt K. Coronary risk in a British Punjabi population: comparative profile of non-biochemical factors. Int $\mathcal{F}$ Epidemiol 1994;23:28-37.

7 Bhopal R, Unwin N, White M, et al. Heterogeneity of coronary heart disease risk factors in Indian, Pakistani, Bangladeshi and European origin populations: cross sectional study. BMF 1999;319:215-20.

8 Khattar RS, Swales JD, Senior R, et al. Racial variation in cardiovascular morbidity and mortality in essential hypertension. Heart 2000;83:267-71.

9 Senior P, Bhopal R. Ethnicity as a variable in epidemiological research. BMF 1994;309:327-30.

10 Nazroo JY. The health of Britain's ethnic minorities. London: PSI; 1997.

11 Kooner JS. Coronary heart disease in UK Indian Asians: the potential for reducing mortality. Heart 1997;78:530-2.

12 Barker DJP. Fetal origins of coronary heart disease. BMF 1995;311:171-4.

13 Bhatnagar D, Anand IS, Durrington PN, et al. Coronary risk factors in people from the Indian subcontinent living in West London and their siblings in India. Lancet 1995;345:405-9.

\section{STAMPS IN CARDIOLOGY}

\section{World Health Organization}
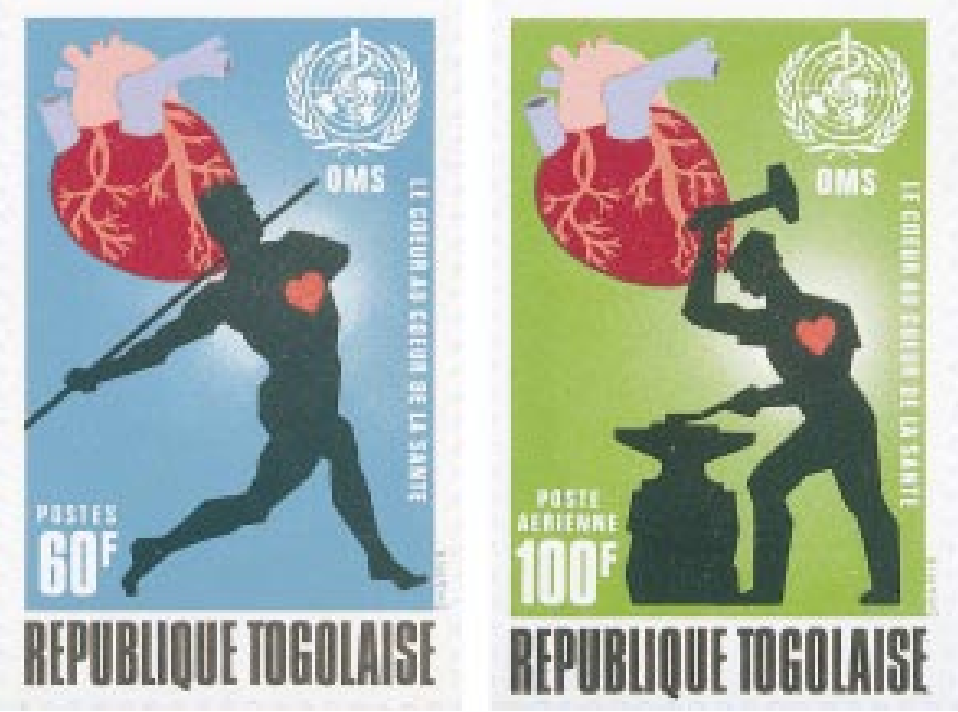

These two stamps from Togo were part of the set commemorating the 1972 World Health Organization campaign "Your heart is your health". Both the anatomical and stylised hearts are incorporated in the designs which have been selected to illustrate fitness, strength and health. A former French territory, the campaign slogan is inscribed in French. Each stamp bears the WHO logo also in French (OMS-Organisation Mondiale de la Sante).

M K DAVIES A HOLLMAN 